\title{
EVOLUTION OF CATACLYSMIC BINARIES
}

\author{
BOHDAN PACZYŃSKI \\ Department of Astrophysics \\ 124 Peyton Hall \\ Princeton University \\ Princeton, NJ 08544, USA
}

\begin{abstract}
The minimum period of hydrogen rich cataclysmic binaries at about 80 minutes is due to evolution driven by gravitational radiation. The nature of the gap between 2 and 3 hour periods is less clear. Magnetic braking may be responsible, but this would be in contradiction with the hibernating nova scenario. It is interesting that contact binaries of W UMa type are subject to little or no magnetic braking.
\end{abstract}

The origin and evolution of cataclysmic binaries has been revied recently by King (1988). Here I shall discuss a few problems that are either new or controversial.

1

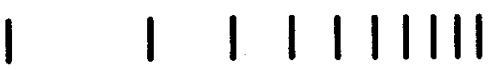

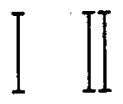

$I$
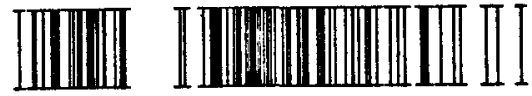

$\prod \amalg$
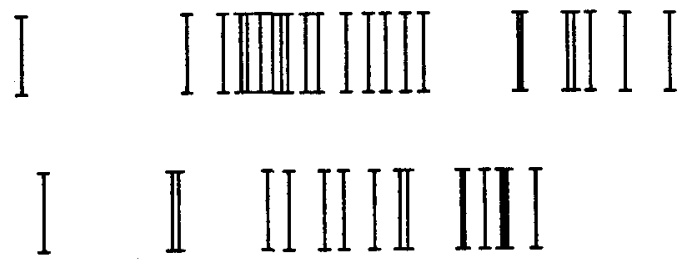

IIII

Figure 1. The distribution of binary periods is shown in a form of three spectra: for cataclysmic variables (upper), for low-mass X-ray binaries (middle), and for related systems (lower) following Ritter (1987). The comparison spectra (top and bottom) are labeled with the binary period (in hours). Four cataclysmic variables with periods below 80 minutes are hydrogen deficient. 
One of the important observations that theory has to explain is the distribution of binary periods. It is presented in Figure 1 as a "spectrum" of periods, each system shown as a vertical line. The cut-off in the distribution of periods of cataclysmic binaries near 80 minutes is understood in terms of evolution driven by gravitational radiation (Paczynski 1981, Paczy ński and Sienkiewicz 1981, Rappaport, Joss and Webbink 1982). The origin of the gap between 2 and 3 hours is less clear. The most popular explanation is binary evolution driven by magnetic braking (Eggleton 1976, Verbunt and Zwaan 1981, Spruit and Ritter 1983, Verbunt 1984, and many others). In order to have a gap between 2 and 3 hours, systems above the gap must be transferring mass at a rate between $2 \times 10^{-9}$ and $10^{-8} M_{\odot} y^{-1}$ (Paczyński and Sienkiewicz 1983, Verbunt 1984, Hameury et al. 1987), more or less as indicated by the observations (Patterson 1984).

The most serious problem with this high mass transfer rate is that accretion of hydro gen rich matter onto a white dwarf primary leads to mild shell flashes, not to nova explosions (Kutter and Sparks 1980, Prialnik et al. 1982, Fujimoto 1982). A very attractive solution to this problem was proposed by Shara and his associates (Shara et al. 1986, Prialnik and Shara 1986, Shara 1988). They find that very old novae, like WY Sge (Nova 1783) and CK Vul (Nova 1670) are 10 to 100 times fainter than the more recent "old novae". They propose that between the eruptions novae are "hibernating", with mass transfer rate and luminosity reduced by orders of magnitude. The proposed space density of such objects is about $10^{-4} p c^{-3}$, i.e. one out 1000 nearby $G, K$, or $M$ dwarfs should be a hibernating nova. The time averaged mass transfer rate is reduced to about $10^{-10} M_{\odot} y r^{-1}$, and hydrogen ignition on the accreting white dwarf is explosive.

It is well established that mass transfer in all kinds of semidetached binaries changes on various time scales. Among cataclysmic variables this is well known for polars, i.e. AM Her type objects (Liebert and Stockman 1985 and references therein). The observed spin-up times for DQ Her type systems are $2 \times 10^{6}$ years (DQ Her), and $3 \times 10^{5}$ years (H2252-035) (Lamb and Patterson 1983). This is much shorter than the expected life time of any cataclysmic binary, and indicates that the currently observed mass transfer rates are higher than average. Notice, that if there are large fluctuations in the mass transfer rate then the accreted nuclear fuel is likely to ignite during the high mass transfer episode, no matter what the reason for the fluctuations is.

There are very interesting and far reaching consequences of the hiberoation hypothesis, at least in its extreme form mentioned above. The average mass transfer rate of only $10^{-10} M_{\odot} y r^{-1}$ requires angular momentum loss at the level provided by gravitational radiation alone. There is no need for magnetic braking. In this case, a new explanation is needed for the period gap between 2 and 3 hours.

Of course, there was never direct evidence that magnetic braking actually operates efficiently in cataclysmic binaries. One should notice that magnetic braking is much less efficient in the contact systems of W UMa type than it is among their predecessors, as the contact systems are so much more numerous than their detached progenitors in the field (Ruciński 1985, Ruciński and Seaquist 1988), as well as in NGC 188 (Kałużny and Shara 1987, Kałuźny 1988). The activity related to surface magnetic fields is smaller in W UMa systems than expected for stars with such short rotation periods (Rucinski 1985). Therefore, it is not obvious why the magnetic braking scenario, based on single or detached systems, should work for semidetached cataclysmic binaries any better than for contact W UMa stars. The origin of the gap remains unclear. 
The issue of what fraction of their lifetimes novae are hibernating is likely to be resolved observationally, by systematic search for short period binaries among nearby G, $\mathrm{K}$, and $\mathrm{M}$ dwarfs. Such searches can be conducted in at least two ways: with photoelectric radial velocity measurements using technique developed by Griffin (1988 and references therein) and also adopted by Mayor (1980, CORAVEL), and with accurate CCD photometry. The expected radial velocity amplitudes are in the range $100-300 \mathrm{~km} \mathrm{~s}^{-1}$, while ellipsoidal light variations of the secondary components filling up their Roche lobes are in the range of $0.1-0.2 \mathrm{mag}$. Binary periods from 1 to 10 hours are expected.

Patterson (1984) noticed that the death rate of novae exceeds by an order of magnitude the birth rate of short period cataclysmic binaries. This discrepancy is even larger if most novae hibernate. If the discrepancy is real then there must be a process that destroys cataclysmic binaries. One may envision a scenario where any given cataclysmic binary transfers only a fraction of the secondary's mass during its life time. In this picture cataclysmic variables would be born at all binary periods, and would spend most of their rather short life close to that period. The gap between 2 and 3 hours may indicate that systems in that range are most prone to self-destruction. Run-away angular momentum loss during a very long and slow nova eruption may be responsible (MacDonald 1986).

It is not likely that theory alone can answer the question of whether a typical cataclysmic variable evolves over a large or small range of binary periods. In particular, do binaries evolve from above the gap to below the gap? It is most likely that large surveys of nearby stars will determine the distribution of hibernating as well as detached binaries, and will help to resolve this issue.

Apart from the gap in their period distribution there are two unusual groups of cataclysmic binaries. There are 7 systems with periods between 0.0788 and 0.0798 days, and 6 systems in the range $0.1396-0.1406$ days. 40 binaries are known to have periods between 80 minutes and 2 hours, i.e. between 0.0556 and 0.0833 days. Assuming uniform distribution the a priori probability of finding 7 systems with periods within 0.001 days anywhere between 80 minutes and 2 hours is 0.02 . There are 34 binaries known with periods between 3 and 4 hours, i.e. between 0.125 and 0.1667 days. For a uniform distribution the probability of finding 6 systems with periods within 0.001 days some where between 3 and 4 hours is 0.02 . These two groups may be just due to chance.

Hameury et al. $(1988 \mathrm{a}, 1988 \mathrm{~b})$ proposed that the group of 6 AM Her type binaries with periods near 0.079 days may be explained within a magnetic braking evolutionary scenario. In order to explain the narrowness of the period range for this group they had to assume that the rate of angular momentum loss was identical for all systems, to within $1 \%$ or so. Considering the complicated nature of stellar magnetic fields and stellar winds, such standardization would be most extraordinary and very important. It will be interesting to see if the grouping persists when the number of known short period cataclysmic binaries has doubled.

The nature of the 2 to 3 hour gap is not clear for cataclysmic binaries, but its existence is beyond any doubt. The low mass X-ray binaries seem to have a cut-off at about 3 hours, but its reality is not so obvious (cf. Figure 1). In any case, there are bydrogen rich systems with periods below 4 hours (UY Vol, Schmidtke and Cowley 1987, GR Mus, Motch et al. 1987), in spite of the theoretical prediction that none should exist below 5 hours (Phinney et al. 1988). One system, Cyg X-3, increases its binary period of 
4.8 hours on a time scale of only $5 \times 10^{5}$ years, making it a likely candidate for a progenitor of the recently discovered eclipsing millisecond pulsar 1957+20 (Fruchter et al. 1988, Molnar 1988). It is not clear what process drives this rapid evolution or whether it applies to other low mass X-ray binaries. The existence of "on" and "of" states and of various types of transient and "X-ray nova" phenomena makes the picture even more confusing. It is impossible at this time to say anything definite about time-averaged mass transfer rates or the variety of evolutionary scenarios for low mass X-ray binaries.

There is another very confusing issue related to cataclysmic binaries and to progenitors of Type I supernovae. In one recent search for short period double white dwarfs, none was found in a sample of 44 (Robinson and Shafter 1987), while other groups found some short period pairs of white dwarls (Saffer, Liebert and Olszewski 1988, Bragaglia et al. 1988). It is not clear what fraction of all white dwarfs is double or whether they are good candidates for the progenitors of Type I supernovae (Tutukov and Yungelson 1979, Webbink 1979, Iben and Tutukov 1984, Paczyński 1985). If future searches establish that the fraction of double white dwarfs is small, the most likely explanation will be a shortage of progenitors for such systems, i.e. main sequence binaries with periods of few years and mass ratio close to one. A survey of about 80 late B-type binaries by Wolff (1978) has revealed only one such system. However, the components of long period binaries are likely to be rapid rotators, so it may be very difficult to discover low amplitude radial velocity variations. Considering all the uncertainties, the deficiency of short period double white dwarfs is not alarming for the theory of evolution of close binaries, but it may be uncomfortable for those who seek the progenitors of Type I supernovae.

It is a great pleasure to acknowledge many discussions with Dr. Virginia Trimble. This project was supported in part by the NSF Grant AST87-18432.

\section{References}

Bragaglia, A., Greggio, L., Renzini, A., and D'Odorico, S. 1988, The Messenger, No. 52, p. 35.

Eggleton, P. P. 1976, IAU Symp. 73, p. 209, Eds: P. P. Eggleton, S. Mitton, and J. A. J. Whelan, (D. Reidel Publ. Co.).

Fruchter, A. S., Stinebring, D. R., and Taylor, J. H. 1988, Nature, 333, 237.

Fujimoto, M. Y. 1982, Ap. J., 257, 767.

Griffin, R. F. 1988, The Observatory, 108, 90.

Hameury, J. M., King, A. R., Lasota, J. P., and Ritter, H. 1987, Ap. J., 316, 275.

Hameury, J. M., King, A. R., Lasota, J. P., and Ritter, H. 1988a, Mon. Not. R. astr. Soc. 231, 535.

Hameury, J. M., King, A. R., and Lasota, J. P. 1988b, Astron. Ap., 195 , L12.

Iben, I. Jr., and Tutukov, A. V. 1984, Ap. J. Suppl., 54, 535.

Kałużny, J. 1988, private communication.

Kałużny, J., and Shara, M. M. 1987, Ap. J., 314, 585.

King, A. R. 1988, Quarterly J. R. A. S., 29, 1.

Kutter, G. S., and Sparks, W. M. 1980, Ap. J., 239, 988. 
Lamb, D. Q., and Patterson, J. 1983, IAU Coll. No 72, p. 229, Eds: M. Livio and G. Shaviv, (D. Reidel Publ. Co.).

Liebert, J., and Stockman, H. S. 1985, in Cataclysmic Variables and Low-Mass X-Ray Binaries, p. 151, Eds: D. Q. Lamb and J. Patterson, (D. Reidel Publ. Co.).

MacDonald, J. 1986, Ap. J., 305, 251.

Mayor, M. 1980, Astron. Ap., 87, L1.

Molnar, L. A. 1988, Ap. J. (Letters), 331, L25.

Motch, C., Pedersen, H., Beuermann, K., Pakull, M. W., and Courvoisier, T. J.-L. 1988, Ap. J., 313, 792 .

Paczyński, B. 1981, Acta Astron., 311.

Paczyński, B., and Sienkiewicz, R. 1981, Ap. J. (Letters), 248, L27.

Paczyński, B., and Sienkiewicz, R. 1983, Ap. J., 268, 825.

Paczyński, B. 1985, in Cataclysmic Variables and Low-Mass X-Ray Binaries, p. 1, Eds: D. Q. Lamb and J. Patterson, (D. Reidel Publ. Co.).

Patterson, J. 1984, Ap. J. Suppl., 54, 443.

Phinney, E. S., Evans, C. R., Blandford, R. D., and Kulkarni, S. R. 1988, Nature, 333, 832.

Prialnik, D., Livio, M., Shaviv, G., and Kovetz, A. 1982, Ap. J., $257,312$.

Prialnik, D., and Shara, M. M. 1986, Ap. J., 311, 172.

Rappaport, S., Joss, P. C., and Webbink, R. F. 1982, Ap. J., 254, 616.

Ritter, H. 1987, Astron. Ap. Suppl., 70. 335.

Robinson, E. L., and Shafter, A. W. 1987, Ap. J., 322, 296.

Ruciński, S. M. 1985, in Interacting binary stars, p. 113, Eds: J. E. Pringle and R. A. Wade, (Cambridge University Press).

Ruciński, S. M., and Scaquist, E. R. 1988, A. J., 95, 1837.

Saffer, R. A., Liebert, J., and Olszewski, W. 1988, Ap. J., 334, ...

Schmidtke, P. C., and Cowley, A. P. 1987, Astron. J., 93, 374.

Shara, M. M. 1988, 20th General Assembly, IAU, Joint Discussion II, this volume.

Shara, M. M., Livio, M., Moffat, A. F. J., and Orio, M. 1986, Ap. J., 311, 163.

Spruit, H. C., and Ritter, H. 1983, Astron. Ap., 124, 267.

Tuiukov, A. V., and Yungelson, L. R. 1979, Acta Astron., 29, 665.

Verbunt, F. 1984, Mon. Not. R. astr. Soc., 209, 227.

Verbunt, F., and Zwaan, C. 1981, Astron. Ap., 100, L7.

Webbink, R. E. 1979, in White Dwarfs and Variable Degenerate Stars, Ed.: H. M. Van

Horn and V. Weidemann (New York: University of Rochester Press), p. 426.

Wolf, S. C. 1978, Ap. J., 222, 559. 